\title{
Granular flow around the horizontal tubes of a particle heat exchanger: DEM-simulation and experimental validation
}

\author{
Philipp Bartsch ${ }^{\mathrm{a}}$, Stefan Zunft ${ }^{\mathrm{b}}$
}

\begin{abstract}
${ }^{a}$ Institute of Engineering Thermodynamics, German Aerospace Center, Pfaffenwaldring 38-40, Stuttgart, Germany, Phone: 49-711-6862753, e-mail: Philipp.bartsch@dlr.de (corresponding author)

${ }^{\mathrm{b}}$ Institute of Engineering Thermodynamics, German Aerospace Center, Pfaffenwaldring 38-40, Stuttgart, Germany, Phone: 49-711-6862601, e-mail: stefan.zunft@dlr.de
\end{abstract}

\begin{abstract}
Moving bed heat exchangers are a central element in solar thermal power plants using granular heat transfer fluids. Their performance is determined by a complex granular flow field in the device. This calls for accurate simulation models forming the basis for novel design tools.

In this study we propose a discrete element model (DEM) to calculate the dense granular flow field around the horizontal tubes of a moving bed heat exchanger.

The simulation results are compared to measurement data using particle image velocimetry (PIV). In the upper part of the tube the model agrees well with the experiment, capturing the relevant flow phenomena like the stagnant zone on top of the tube.

Below the tube, a void area is observed both in the experiment and the simulation. However, the PIV analysis of the experimental data doesn't resolve the void area and the associated flow phenomena due to the highly dynamic flow state, the inherent nature of PIV technique and additional disturbing effects in the setup. Therefore, the model can't be validated in the corresponding regions.

Still, the deviations between simulation and experiment are confined to a very narrow region directly at the tube surface in the lower part of the tube. In the rest of the flow field, the model proves to be a reliable tool for further investigations and will be used in future studies.
\end{abstract}

\section{Introduction}

Granular materials are still a challenge to physical and mathematical description [1]. They may behave like a solid, e.g. like vacuum-packed coffee powder, or similar to a liquid, e.g. like an hour glass. This dualistic behavior in combination with other complex phenomena leads to challenges regarding a precise prediction of dense granular flows. On the other hand granular materials are omnipresent in everyday life and play a crucial role in many industrial processes.

An application which recently has gained growing attention is to use granular materials, such as sand or bauxite proppants, as a heat transfer medium in solar thermal power plants [2]. The particles are heated up by concentrated solar irradiance and are stored in a thermally insulated tank. Subsequently, in a particle steam generator the thermal energy of the bulk material is used to fuel a power plant cycle to generate electricity. A favorable design option of such a particle steam generator is a moving bed heat exchanger (MBHE) with horizontally arranged tubes. Compared to plates, such a tubular heat exchanger design is advantages regarding the high operating pressures of the secondary fluid, occurring in power plant applications. Furthermore, MBHE benefit from low parasitic loads as the granular material doesn't have to be fluidized.

Heat transfer between granular materials and immersed surfaces has been investigated for several decades (e.g. [3] [4] [5] [6]). Several experimental works also exist concerning the heat transfer between a moving packed bed and a horizontal tube (cylinder) ( [7] [8] [9] [10] [11]). The residence time of the granular material at the heat transferring surface was found to be a 
key parameter for the heat transfer rate which means that the granular flow field is of major importance for the thermal performance.

The granular flow pattern around horizontally arrange tubes is characterized by a stagnant area at the tube vertex and a void area below the tube. It has primarily been investigated experimentally [12] [7] [8] [13] but there are only a few works concerning the modelling the granular flow [12] [7] [14] [15] in such a geometry. The existing works are either (semi-) empirical models [7], aim at relatively rapidly moving flows [14], or do not capture the characteristic flow phenomena [12]. Lee et al. [15] applied an FEM model but showed no information about the calculated granular flow field at all.

Granular flows in general can be modelled either as a continuum or as discrete particles. Furthermore, different approaches are used depending on the type of granular flow. Continuum models for rapidly moving, dilute and collision-dominated flows generally follow the kinetic theory of granular fluids (KTGF) [16]. Such models, for example, have been applied to fluidized beds [16] [17], chute flows [18] and other rapidly moving systems [14]. Continuum models for dense, slow and friction-dominated granular flows most commonly use plasticity models [19]. Such models have been used to model the flow in hoppers [20], bunkers and bins [21] or in geophysical problems [22].

Discrete particle models are per se a more realistic description of the granular material and no constitutive models are required. These methods tackle the problem on the micro scale by tracking the position and velocity of every single particle in the system. Thus, they provide very detailed insight into the granular assembly, however, at the cost of high computational expense.

Besides the so-called hard sphere models - event-driven methods for dilute flows (e.g. [17]) and "contact dynamics" (CD) for quasi-static systems of rigid particles [23] - the discrete element method (DEM) has become very popular in the last decades. It has been used to investigate the behavior of particulate solids in different fields such as agricultural [24], chemical, pharmaceutical [25] [26] and heavy industries [27]. It has also been applied in thermal energy storage technology to investigate thermally induced stresses in packed-bed heat storage systems [28].

In this work we introduce a DEM model and calculate the granular flow in a moving bed heat exchanger. To validate the model, the simulation results are compared to measurements using particle image velocimetry (PIV).

\section{Experimental setup}

The moving bed heat exchanger test infrastructure used for this study is shown in FIGURE 2 (left) and has been described in detail in [29]. It basically consists of a storage container for the granular material, a particle heat exchanger and a conveyor device. From the storage tank the material flows, driven by gravity, through the test section where arbitrary heat exchanger geometries can be inserted. The chain conveyor returns the material back from the heat exchanger outlet into the storage container.

The heat exchanger test rig is filled with quartz sand of an average (median) grain size of $d_{\mathrm{p} 50} \approx 0.6 \mathrm{~mm}$ (cf. FIGURE 1). The average flow speed in the free cross-section of the heat exchanger is evaluated from the mass flow rate of the tube chain conveyor and the bulk density and we obtain $u_{\text {ref }} \approx 4 \mathrm{~mm} / \mathrm{s}$. 


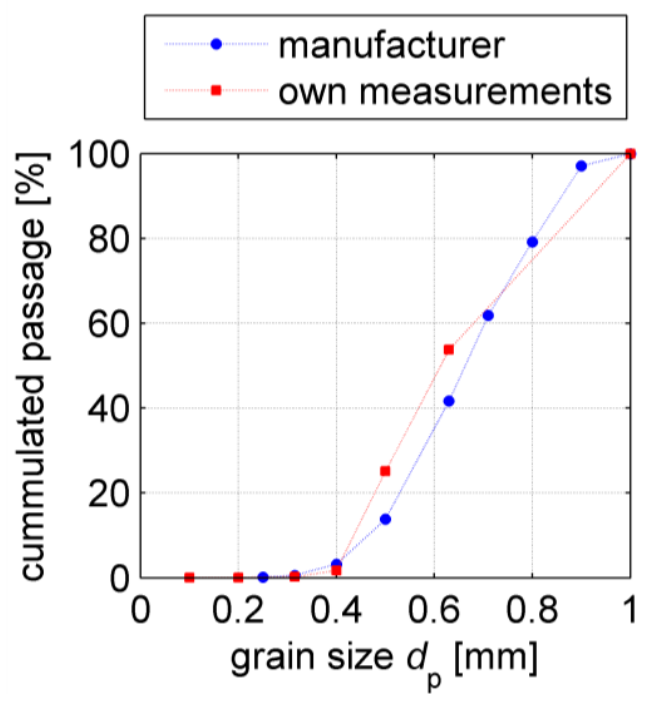

FIGURE 1. Grain size distribution of the used quartz sand (Median grain size $d_{\mathrm{p} 50} \approx 0.6 \mathrm{~mm}$ ).

To allow a visual inspection of the bulk flow, the heat exchanger is replaced by an acrylic glass mock-up shown in FIGURE 2 (middle). When operated, the bulk material enters at the top and flows around the tubes.

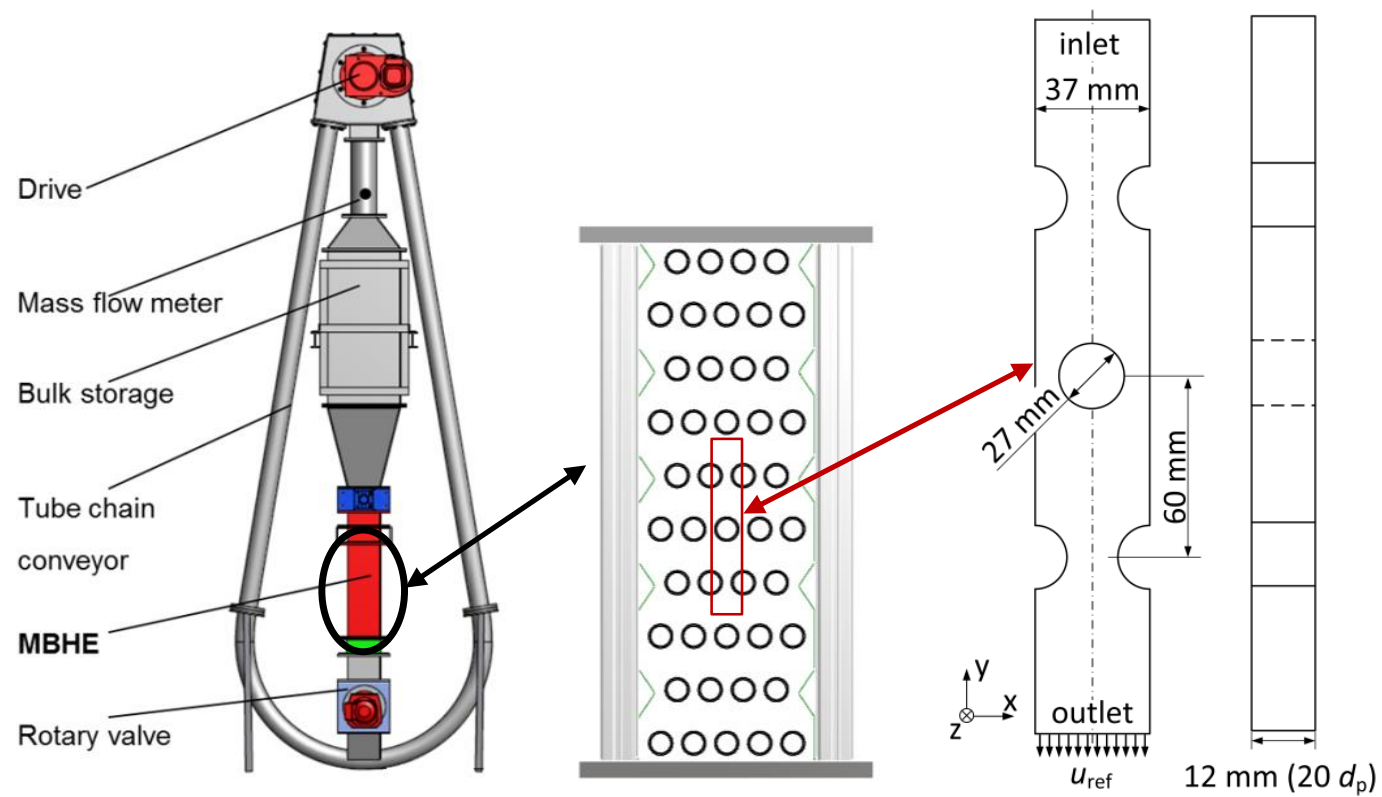

FIGURE 2. Moving bed heat exchanger test rig (left). Heat exchanger mockup (middle). Simulation geometry (right).

We examine the granular flow inside the mockup by means of particle image velocimetry (PIV). It is based on the analysis of successive images of the flow. The images are subdivided by a grid of stationary control volumes. By detecting recurring patterns and determining their displacement in a pair of images the local velocity of the flow can be calculated. Unlike for fluids, in granular flows no extra tracer particles are needed as the patterns are formed by the grains themselves. A more detailed description of PIV in the context of granular flows can be found elsewhere [30] [31].

Pictures are taken at a rate of $25 \mathrm{~Hz}$ through the transparent acrylic glass front of the mockup. To reduce temporal fluctuations of the local flow speed we average the results over a sequence of 40 pairs of images. The size of the PIV control volumes is $\Delta x=0.6 \mathrm{~mm}$ and $\Delta y=1.6 \mathrm{~mm}$. The size of the control volumes was chosen according to the flow speed to be expected. The 
displacement between two images should be about a quarter of the size of the control volumes [32]. As the flow speed in $y$-direction is higher than in $x$-direction, $\Delta y$ is greater than $\Delta x$.

This resolution is very fine compared to conventional PIV analysis using tracer particles and requiring $10 \ldots 25$ particles per CV [32]. However, in our case the images are rich in contrast and the PIV-algorithm doesn't necessarily track the particles but rather an optical structure in the image.

\section{Numerical model}

We use a discrete particle model to simulate the granular flow in a representative section of the heat exchanger mockup. The simulation geometry is given in FIGURE 2 (right). In x-direction we use periodic boundary conditions. In z-direction the geometry is 20 particle diameters thick and is confined by walls. The specific focus is on the simulation of the flow field close to the the acrylic glass front wall as this is the area which is captured by the experiment. Therefore, the surface friction between bulk and acrylic glass at the front wall is considered in the model. At the rear wall surface friction is set to zero.

At the bottom of the geometry we set the outlet velocity $u_{\text {ref }}$ by a moving horizontal plate (face normal parallel to $y$-axis). The plate moves into vertical direction dragging particles down with it. Particles are generated at a small distance above the inlet such that the simulation domain is always filled with particles.

The modelling approach we use in this work was first introduced by Cundall and Strack [33] and is known in literature as Discrete Element Method (DEM). The method is based on solving the Newtonian equations of motion for each particle for translational and rotational motion.

$$
\sum_{k} \boldsymbol{F}_{i, k}=m_{i} \ddot{\boldsymbol{x}}_{i}
$$

$$
\sum_{k} \boldsymbol{M}_{i, k}=J_{i} \dot{\boldsymbol{\omega}}_{i}
$$

The acting forces and moments include body forces such as the gravitational force as well as external forces and moments which for example may originate from contacts with other particles and walls.

The contact forces are calculated according to the so-called soft sphere model which is based on the evaluation of a (virtual) overlap between particles or particles and surfaces. When solving the equations of motion, two grains may turn out to overlap due to the finite time step. This overlap is interpreted as the elastic deformation which occurs for particles under stress [34].

\subsection{Contact models}

Several contact models have been developed to translate this elastic deformation into a normal interaction force between particles [35]. Here, we use a linear hysteresis model which first has been introduced by Walton and Braun [36]. The instantaneous normal interaction force basically is calculated as follows:

$$
F_{n}^{t}= \begin{cases}\min \left(F_{n}^{t-\Delta t}+k_{u l} \Delta s_{n}, k_{l} s_{n}\right), & \Delta s_{n} \geq 0 \\ \max \left(F_{n}^{t-\Delta t}+k_{u l} \Delta s_{n}\right), & \Delta s_{n}<0\end{cases}
$$

The model distinguishes between loading case $\left(\Delta s_{\mathrm{n}} \geq 0\right)$ and unloading case $\left(\Delta s_{\mathrm{n}}<0\right)$ where $\Delta s_{n}=\left(s_{n}^{t}-s_{n}^{t-\Delta t}\right)$ is the change in normal overlap between two time steps. Furthermore, it is distinguished between a loading and unloading normal contact stiffness $k_{1}$ and $k_{\mathrm{ul}}$, respectively, which are related to each other by the coefficient of restitution $e$ :

$$
e=-\sqrt{k_{l} / k_{u l}}
$$


The contact stiffness for the loading case $k_{1}$ is calculated by material parameters:

$$
k_{l}=\frac{E_{1} d_{p, 1} \cdot E_{2} d_{p, 2}}{E_{1} d_{p, 1}+E_{2} d_{p, 2}}
$$

Here, index " 1,2 " denote the two elements forming the considered contact, which might either be a particle-particle-contact or a particle-wall-contact. $d_{\mathrm{p}}$ is the particle diameter and $E$ is the Young's Modulus of the contacting elements [37].

The tangential interaction forces are calculated according to the following elastic-frictional force model:

$$
\boldsymbol{F}_{t}^{t}=\frac{\boldsymbol{F}_{t}^{t}}{\left|\boldsymbol{F}_{t}^{t}\right|} \begin{cases}\min \left(\left|\boldsymbol{F}_{t}^{t-\Delta t}+k_{l} \Delta \boldsymbol{s}_{t}\right|, \mu_{s} F_{n}^{t}\right), & \text { if no sliding occurs } \\ \min \left(\left|\boldsymbol{F}_{t}^{t-\Delta t}+k_{l} \Delta \boldsymbol{s}_{t}\right|, \mu_{d} F_{n}^{t}\right), & \text { if sliding occurs }\end{cases}
$$

As one can see from eq. (6) the tangential contact force $\boldsymbol{F}_{t}^{t}$ between two contacting elements evolves with the relative tangential displacement at the current time $\Delta \boldsymbol{s}_{\mathrm{t}}$.

Sliding occurs, if $F_{t}^{t}$ exceeds the limit of $\left(\mu_{s} \cdot F_{n}^{t}\right)$, with $\mu_{\mathrm{s}}$ being the static friction coefficient. Once $F_{t}^{t}$ falls below the value of $\left(\mu_{d} \cdot F_{n}^{t}\right)$, with $\mu_{\mathrm{d}}$ being the dynamic friction coefficient.

For a reduced computational expense we assume spherical particles in the simulations. Unfortunately, with this simplification the model loses its ability to predict the high resistance of non-spherical particles against a rolling motion. A common way to remedy this deficit is to introduce a resistive torque applied to contacting particles. The concept is known as "rolling friction" [38], and different classes of rolling friction models have been introduced. In this work we use an elastic-plastic spring dashpot model, commonly referred to as "Model C" [38], using a "rolling friction coefficient", $\mu_{\mathrm{r}}$, as an input parameter.

The resistive torque $\boldsymbol{M}_{r}^{t}$ (at time $t$ ) is defined as follows [37]:

$$
\boldsymbol{M}_{r}^{t}=\min \left(\left|\boldsymbol{M}_{r, e}^{t}\right|, M_{r, l i m}\right) \frac{\boldsymbol{M}_{r, e}^{t}}{\left|\boldsymbol{M}_{r, e}^{t}\right|}
$$

$M_{r, \text { lim }}$ is a limiting value of the torque, depending on the normal force $F_{n}$, the rolling resistance (rolling friction) coefficient $\mu_{\mathrm{r}}$, and the rolling radius $R_{r}$ :

$$
M_{r, l i m}=\mu_{r} R_{r} F_{n}
$$

In case of mono-sized, spherical particles the rolling radius is $R_{\mathrm{r}}=d_{\mathrm{p}} / 2$. The rolling friction parameter $\mu_{\mathrm{r}}$ can be interpreted as the tangent of the maximum angle of a slope on which the rolling resistance moment counterbalances the moment produced by gravity in the particle. $\mu_{\mathrm{r}}$ is usually calibrated from experimental data as shown in the following section.

Below the limiting value $M_{r, l i m}$, the resistive torque is allowed to vary continuously according to a linear elastic model:

$$
\boldsymbol{M}_{r, e}^{t}=\boldsymbol{M}_{r}^{t-\Delta t}-k_{r} \boldsymbol{\omega}_{r e l} \Delta t
$$

$\boldsymbol{M}_{r}^{t-\Delta t}$ is the resistive torque at the previous time step, $\boldsymbol{\omega}_{\text {rel }}$ is the relative angular velocity between the two contacting particles (wall and particle) and $k_{\mathrm{r}}$ is the 'rolling stiffness':

$$
k_{r}=R_{r}^{2} k_{l}
$$


Due to the elastic part $\boldsymbol{M}_{r, e}^{t}$ of the resistive torque, discontinuities as they occur in other rolling friction models are avoided [38].

\subsection{Model parameterization}

The input parameters of the DEM-model include material parameters such as grain size and Young's modulus as well as material interaction parameters like friction coefficients. According to the experimental setup described in section 2, the grain size is set to $d_{\mathrm{p}}=0.6 \mathrm{~mm}$. The Young's modulus of the particles is set to $E_{\mathrm{p}}=10^{6} \mathrm{~N} / \mathrm{m}^{2}$ which is about three orders of magnitude lower than values of typical materials such as for example sand stone [39].

Using a reduced Young's modulus (reduced contact stiffness) is motivated by increasing the time step of the simulation which scales with the inverse square root of the contact stiffness $\left(\Delta t \sim 1 / \sqrt{k_{l}}\right)$ [37], leading to a substantial saving of computation time. This measure is very common in DEM models [40] and is justified as long as the normal overlap between particles is less than $1 \%$ of the particle radius [26]. In the current case the maximum normal forces between particles are $F_{\mathrm{N}, \max } \approx 0.001 \mathrm{~N}$ which leads to a normal overlap of $0.6 \%$ of the particle radius (cf. eq. (3)). Furthermore, to ensure independence of the simulation results on the contact stiffness, a variation of the Young's modulus was conducted (up to $E_{\mathrm{p}}=10^{8} \mathrm{~N} / \mathrm{m}^{2}$ ) and virtually no impact was found.

In contrast to the contact stiffness, the friction parameters are expected to be a determining for the simulation results. Senetakis et al. [41] investigated the inter-particle coefficient of friction of Leighton Buzzard Sand. They found that the coefficient of dynamic friction and the coefficient of static friction are of very similar magnitude and measured values of $\mu_{\mathrm{pp}}=0.1 \ldots 0.23$. Following their results we set the friction coefficient between particles to $\mu_{\mathrm{pp}}=0.2$.

To deduce the rolling friction coefficient $\mu_{\mathrm{r}}$ we perform a simple laboratory experiment and determine the angle of repose of quartz sand $\varphi_{\mathrm{r}} \approx 34^{\circ}$. In order to reproduce this value in the simulations (with $\mu_{\mathrm{pp}}=0.2$ ) a rolling friction coefficient of $\mu_{\mathrm{r}} \approx 0.3$ has to be adjusted.

The surface friction coefficient between the bulk material and the acrylic glass front wall $\left(\mu_{\text {front }}\right)$ is an important input parameter for the simulation model and has is determined experimentally. We use an annular shear cell to measure the friction coefficient which is dependent of the normal stress between bulk and surface. We estimate the normal horizontal stress at the front wall of the mockup by applying the well-known model developed by Janssen (e.g. in [42]) and find the horizontal stress to be $\sigma_{\mathrm{h}}<1500 \mathrm{~Pa}$. The measured friction coefficient for this normal stress is $\mu_{\text {front }} \approx 0.5$.

The surface friction coefficient at the tube walls has to be estimated. Baumann et al. [30] measured wall friction coefficients of $\mu_{\mathrm{w}}=0.2 \ldots 0.3$ of granular materials on polished stainless steel. Compared to this, the steel tubes in our experimental setup have a relatively rough surface and we assign a higher value in our simulations of $\mu_{\mathrm{w}}=0.4$.

The model parameters are summarized in TABLE 1.

TABLE 1. Simulation parameters

\begin{tabular}{llll}
\hline Parameter & Symbol & Value & Unit \\
\hline Particle diameter & $d_{\mathrm{p}}$ & 0.6 & $\mathrm{~mm}$ \\
Youngs modulus of particles & $E_{\mathrm{p}}$ & $10^{6}$ & $\mathrm{~N} / \mathrm{m}^{2}$ \\
Surface friction coefficient front wall & $\mu_{\text {front }}$ & 0.5 & - \\
Surface friction coefficient rear wall & $\mu_{\text {rear }}$ & 0.0 & -
\end{tabular}




$\begin{array}{llll}\text { Friction coefficient between particles } & \mu_{\mathrm{pp}} & 0.2 & - \\ \text { Rolling friction coefficient } & \mu_{\mathrm{r}} & 0.3 & - \\ \text { Outlet velocity } & u_{\mathrm{ref}} & 4.0 & \mathrm{~mm} / \mathrm{s}\end{array}$

\subsection{Averaging procedure of simulation results}

An adapted post-processing of the DEM-simulation results allows a direct comparison with the results of the PIV-measurements. The DEM basically yields location and velocity of every single particle at a given time. To compare the DEM-simulation results to those of the PIV measurements we have to apply some averaging procedure in space and time to the DEMresults. For this purpose locally fixed control volumes (CVs) have to be defined wherein the averaging is carried out.

Lätzel [34] investigated two different averaging methods to compare their DEM simulations to experimental data of an annular shear cell. His basic averaging formalism for obtaining an averaged quantity $Q$ inside a control volume $V$ is

$$
Q=\frac{1}{V} \sum_{i \in V} w_{i}^{V} V_{i} Q_{i},
$$

with $V_{\mathrm{i}}$ being the particle volume and $Q_{\mathrm{i}}$ being the considered quantity attributed to particle $\mathrm{i}$. In case of the averaged velocity $\left(Q=u_{\mathrm{x}}, Q=u_{\mathrm{y}}\right)$ the quantity is averaged based on the number of particles $N_{\mathrm{i}}$ inside the control volume:

$$
Q=\frac{1}{N_{i}} \sum_{i \in V} w_{i}^{V} Q_{i}
$$

The parameter $w_{i}^{V}$ is the weight of the particles contribution to the average. In this work we use the simplest choice of $w_{i}^{V}$ which is

$$
w_{i}^{V}=\left\{\begin{array}{l}
1, \text { if the center of the particle lies inside the } \mathrm{CV} \\
0, \text { otherwise }
\end{array} .\right.
$$

Lätzel showed that the method is sufficiently precise as long as the diameter of the CV is greater than the particle diameter. Consequently, we choose the size of the CVs in such a way that this requirement is met.

Two different arrangements of the CVs is used depending on the kind of velocity profiles to be analysed. For the evaluation of the horizontal velocity-profiles (FIGURE 4) and the contour plots (FIGURE 3), we arranged the DEM CVs similar to those in the PIV (rectangular CVs, $\Delta x=1.6, \Delta y=1.6 \mathrm{~mm}, \Delta \mathrm{z}=0.6 \mathrm{~mm}$ ). For the evaluation of the velocity at the tube surface (Fig. 4), we arranged them in circular layers around the tube and tangent to the tube surface (CV-size: $0.65 \mathrm{~mm}$ in radial, $1.6 \mathrm{~mm}$ in tangential direction, and $0.6 \mathrm{~mm}$ in axial direction).

\section{Comparison of simulation and experiment}

FIGURE 3 shows contour plots of the granular flow field obtained from the PIV measurement (left) and DEM simulation (right). The tube in experimental plot is located in the center of the mockup (see framed area in FIGURE 2 (middle)). 
While the simulated velocity profile is symmetric to the vertical center line of the tube the experimental profile is slightly asymmetric. Higher velocities are observed on the left half of the plot. There are several possible reasons for this imperfect velocity distribution: The tube chain conveyer which removes the granular material at the bottom of the test rig (cf. FIGURE 2) is a source of unevenness in the flow pattern. Additionally, the surface friction of the acrylic front wall might show slight variations influencing the observable flow. Furthermore, the free cross section of the mock-up slightly changes between two successive rows of tubes due to the alternating number of tubes per row and the guiding plates at the lateral sides of the mockup.

Due to these disturbing effects, the flow profile is not completely even along the horizontal cross section of the mockup.
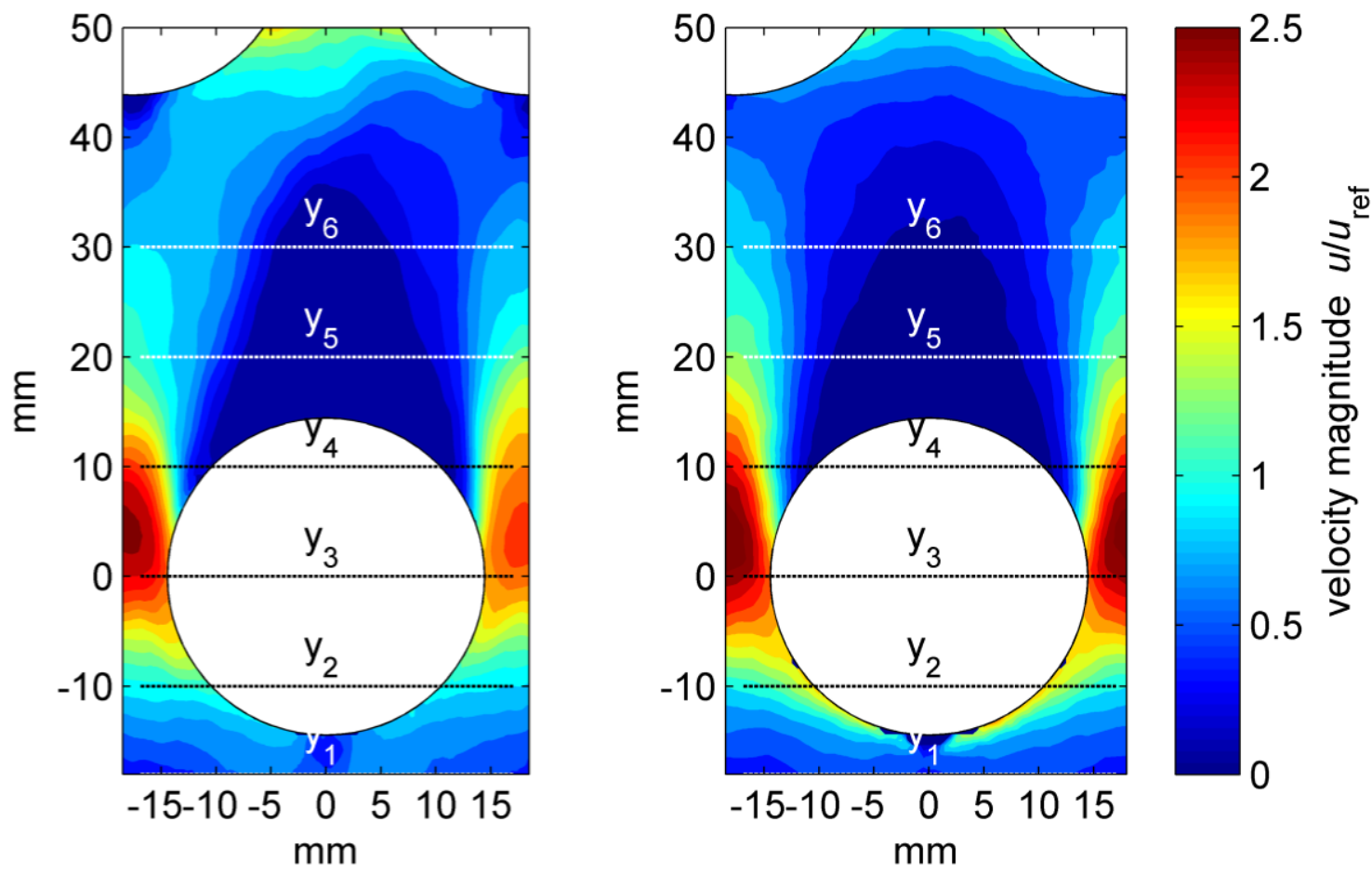

FIGURE 3. Contour plots of granular flow speed. Left: PIV-measurement. Right: DEM-simulation

A cone-shaped stagnant area forms above the tube wherein the flow speed is approximately zero. The stagnant zone is framed by areas of increased particle motion. A continuous transition from the static to the flowing regime is observed. Size and shape of the stagnant area is similar in simulation and experiment. Such stagnant zones have been reported in earlier studies [8] [7] [29]. They are of major importance for the design of moving bed heat exchangers as they hamper the heat transfer from the tube surface to the bulk.

At the lateral sides of the tube the flow velocity increases due to the constriction of the flow cross section. Both plots coherently show the maximum flow speed to occur at $y \approx 5 \mathrm{~mm}$ which is slightly above the center point of the tube $\left(y_{3}=0\right)$. A velocity gradient is observed in radial direction at the tube surface.

In the lower half of the tube we observe a thin layer of elevated velocity directly at the tube surface in the simulation. In the experiment this layer is not observed and the velocity at the tube surface is much lower. This deviation is attributed to the formation of a void area below the tube and will be addressed in more detail in the following section.

FIGURE 4 (a) shows the horizontal velocity profiles of simulation and experiment in the upper half and above of the tube $\left(y_{4}, y_{5}\right.$ and $\left.y_{6}\right)$. Velocities close to zero are observed in the center of the plot where the horizontal profiles intersect the tube $\left(y_{4}\right)$ and the stagnant area above the tube 
$\left(y_{5}, y_{6}\right)$. At the sides of the plot the velocity increases as the horizontal planes reach into the area of increased particle motion.

On the right hand side and in the center of FIGURE 4 (a), simulation and experiment agree well with each other. On the left hand side of the plot (left hand side of the tube) we observe deviations on planes $y_{6}$ and $y_{5}$ in the range of $-14 \mathrm{~mm} \lesssim x \lesssim-7 \mathrm{~mm}$. In this $x$-range the transition from the stagnant to the flowing regime takes place. Due to the horizontal asymmetry in the experimental data, the transition on the left hand side is more distinct than on the right.

(a)

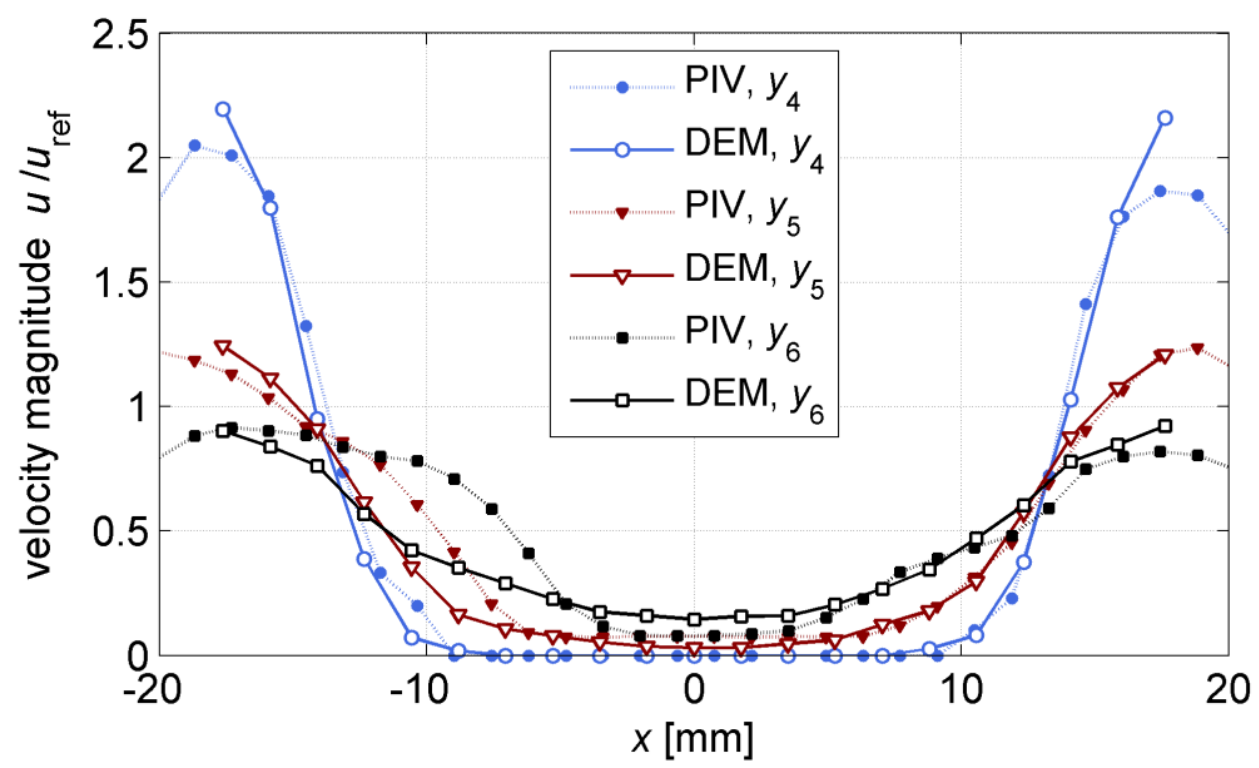

(b)

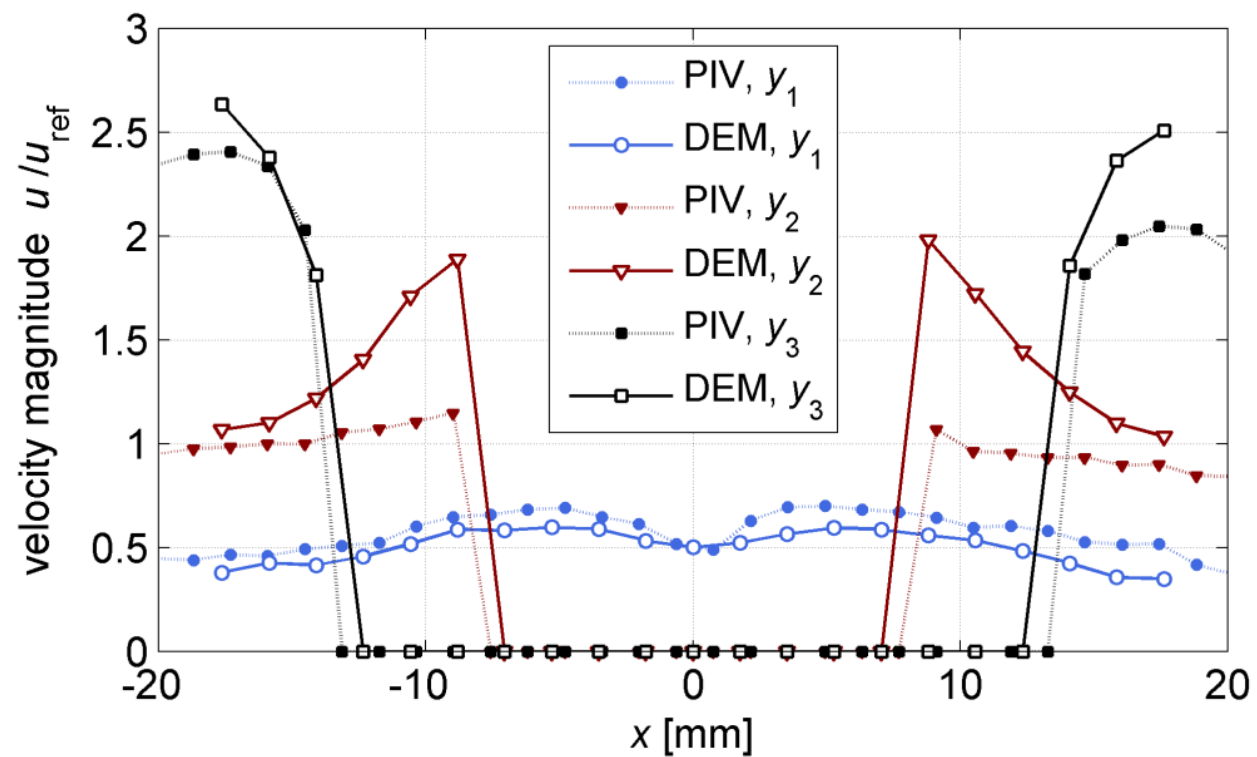

FIGURE 4. Horizontal velocity profiles at different heights (see FIGURE 3). (a) Upper half and above the tube. (b) Lower half and below the tube. Solid lines: PIV-Measurement. Dotted lines: DEM-simulation.

FIGURE 4 (b) shows the horizontal velocity profiles of simulation and experiment in the lower half and below of the tube $\left(y_{1}, y_{2}\right.$ and $\left.y_{3}\right)$. Planes $y_{3}$ and $y_{2}$ intersect the tube and show a jump of the flow speed at the tube surface. Plane $y_{1}$ is located below the tube.

Plane $y_{3}$ again displays the asymmetry of the experimental profile we already mentioned. As a consequence the simulation agrees better on the left than on the right hand of the plot. 
Plane $y_{2}$ intersects the tube surface at $x \approx \pm 9 \mathrm{~mm}$ which is obvious by the jump in flow speed in both plots. At a greater (horizontal) distance from the tube surface $(|x|>16 \mathrm{~mm})$ simulation and experiment yield similar velocity magnitudes. Directly at the surface, however, the flow speed in the simulation is about twice as high as in the experiment. These deviations occur on both sides of the tube and cannot be attributed to the asymmetry of the experimental flow profile. They have already been noticed in the contour plots in FIGURE 3 where we observed a thin layer of elevated flow speed in the lower half of the tube in the simulation but not in the experiment. The reason for the deviation deserves further discussion and will be addressed in detail in the following section.

Plane $y_{1}$ shows a relatively even profile. Both, simulation and experiment, show a small dip in the profile in the center of the plot where the flows from both sides merge below the tube.

The results presented in this section can be summarized as follows:

Deviations between simulation and experiment are primarily attributed to the asymmetry of the measured flow field. As a consequence maximum deviations of up to $60 \%$ of the reference velocity $\left(|\Delta u| / u_{\text {ref }}<0.6\right)$ are observed, but only in specific areas (especially $y_{5}$ (left) and $y_{6}$ (left)).

Furthermore, deviations between simulation and experiment are observed in a very narrow region below the tube where the simulation yields much higher velocity magnitudes than the experiment due to the formation of a void area.

In the rest of the flow field simulation and experiment agree well with each other and the absolute deviations are less than $15 \%$ of the reference velocity $\left(|\Delta u| / u_{\text {ref }}<0.15\right)$

\subsection{Velocity profile at the tube surface}

In this section we compare simulation and experiment based on the velocity profile along the tube surface. The flow pattern close to the tube surface is of major importance for the heat transfer from the tube surface to the bulk.

FIGURE 5 (left) shows the velocity profiles of PIV measurement and DEM simulation along the entire circumference of the tube. The plots denoted by "PIV ${ }_{1.5}$ " and "DEM 1.5 " are profiles at a distance of 1.5 particle diameters $(\approx 0.9 \mathrm{~mm})$ from the tube surface. This is the data closest to the surface we get from the PIV analysis. In addition a third plot is given ("DEM") which shows the velocity profile along the tube surface at a distance of four particle diameters $(\approx 2.4 \mathrm{~mm})$ from the tube wall.

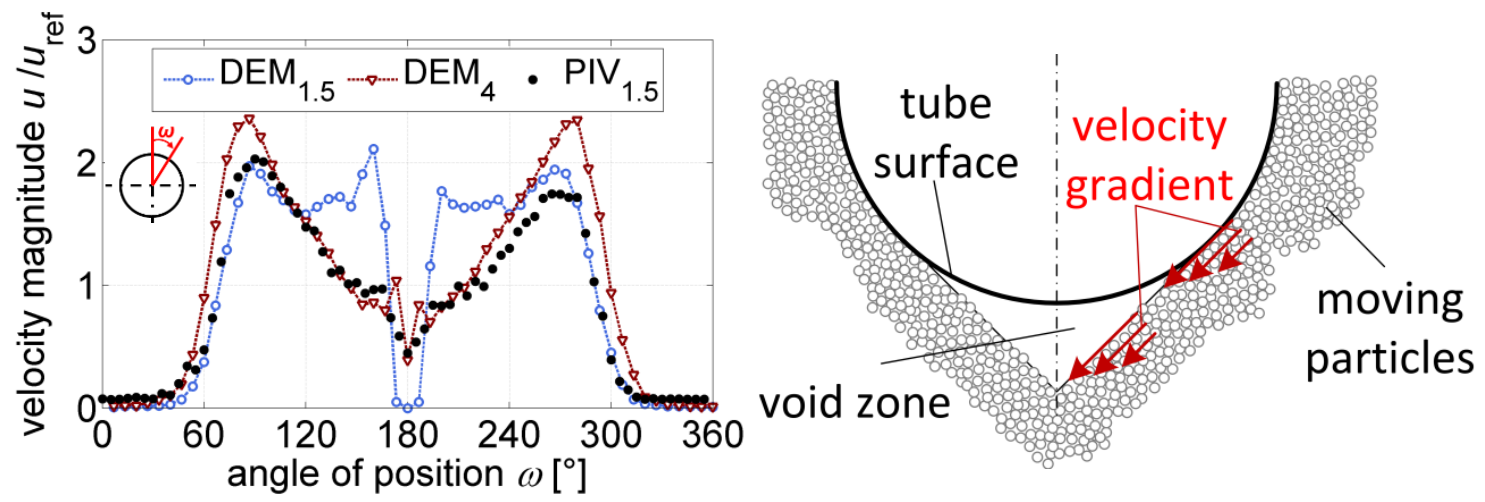

FIGURE 5. Left: Flow speed along the tube surface. $\operatorname{DEM}_{1.5}$ and $\mathrm{DEM}_{4}$ : velocity profiles from simulation at a distance of 1.5 and 4 particle diameters from the tube surface. PIV 1.5 $_{\text {: velocity }}$ profile from measurement at a distance of 1.5 particle diameters from the tube surface. Right: Schematic of the flow pattern below the tube including the velocity gradient at the flanks of the void area.

The stagnant area is located around the tube vertex and we find the flow speed to be close to zero in the range of $\omega \lesssim 50^{\circ}$ and $310^{\circ} \lesssim \omega$ in all three plots. However, the measurement shows slight motion also in the stagnant area $\left(u \approx 0.07 \cdot u_{\mathrm{ref}}\right)$ whereas in the simulation the flow is 
virtually at rest $\left(u \approx 0.005 \cdot u_{\mathrm{ref}}\right)$. At $\omega \approx \pm 50^{\circ}$ from the tube vertex the velocity rises and reaches a maximum at $\omega \approx 90^{\circ}\left(\omega \approx 270^{\circ}\right)$. To this point, simulation and experiment $\left(\mathrm{DEM}_{1.5}\right.$ and $\left.\mathrm{PIV}_{1.5}\right)$ deviate by less than $10 \%$ of the reference velocity $\left(|\Delta u| / u_{\text {ref }}<0.1\right)$

In the lower half of the tube $\left(90^{\circ}<\omega<180^{\circ}\right)$ the plot of $\mathrm{DEM}_{1.5}$ differs significantly from the measured profile PIV $_{1.5}$. The flow speed remains at a high level until it drops to zero at $\omega \approx 160^{\circ}$. In contrast to that, the experimental profile displays a continuous decrease of flow speed until a minimum of $0.5 \cdot u_{\text {ref }}$ is reached directly below the tube. This resembles very much the simulation profile $\mathrm{DEM}_{4}$ at a greater distance from the wall.

These deviations in the lower part of the tube have already been observed in the previous section (cf. $y_{2}$ in FIGURE 4 (b)) and are explained as follows: Below the tube a void zone forms as illustrated in FIGURE 5 (right). In the range of $160^{\circ}<\omega<200^{\circ}$ no particles are found at the tube surface and hence the velocity is zero. In the range of $120^{\circ}<\omega<160^{\circ}\left(200^{\circ}<\omega<240^{\circ}\right)$ the particles gradually lose touch with the tube surface before the actual void area is formed. As a consequence the decelerating impact of the wall friction decreases and the particles start to accelerate along the slope.

The void area as well is visible in the experimental setup and we also observe particles moving down the slope very fast in the raw measurement data (raw images, see FIGURE 6).

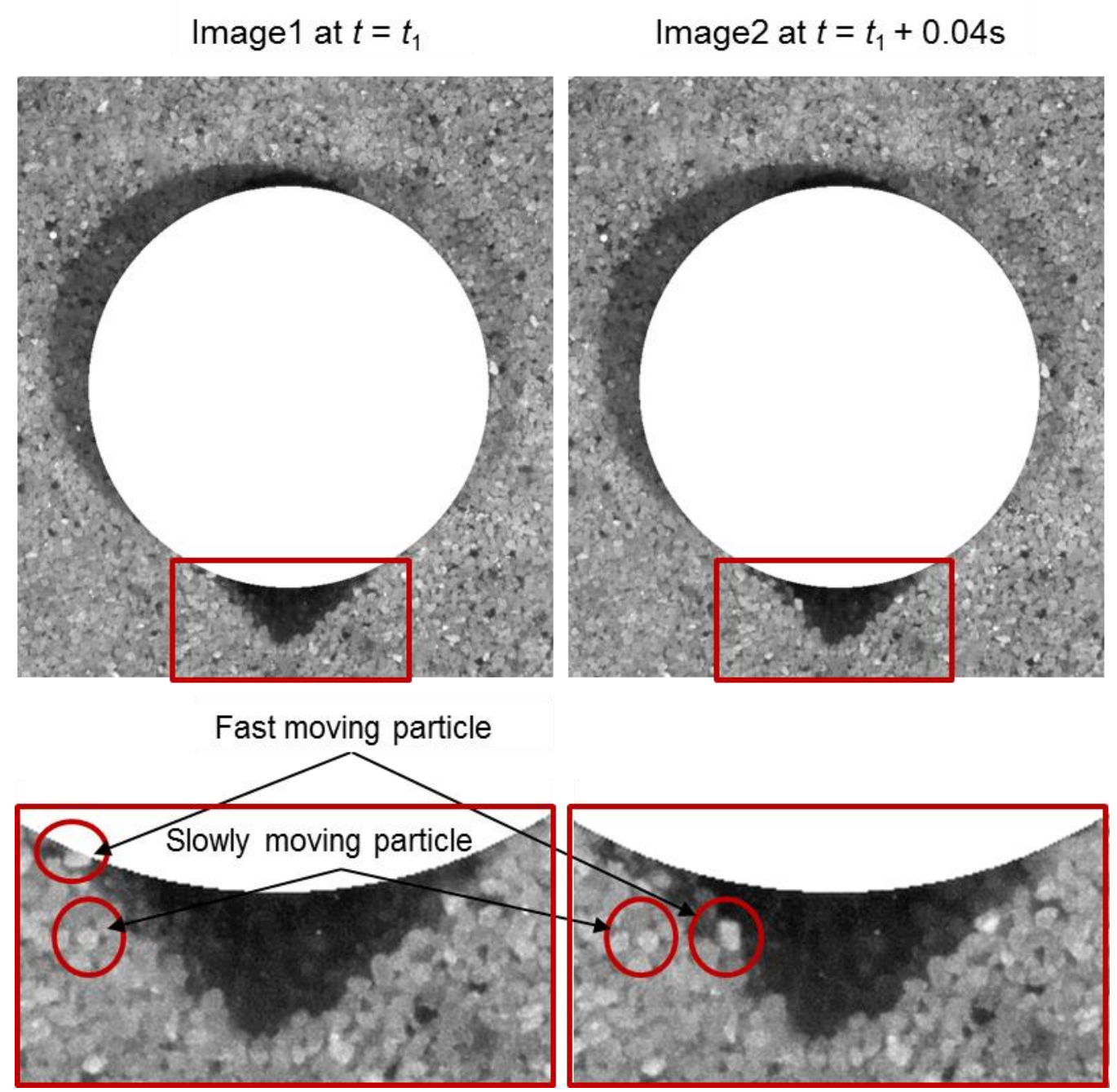

FIGURE 6. Example of two (successive) raw images used for PIV-measurement.

However, the subsequent PIV analysis doesn't capture the effects due to the following reasons: 
- In a control volume which is being intersected by the flanks of the void zone the PIV yields an average velocity of the "zero-velocity" of the void and the elevated flow speed of the particles. In contrast to that, the simulation results are averaged based on the particle number (cf. eq. (12)). Therefore, the PIV yields lower velocities than the simulation at the edges of the void area.

- The camera partly looks into the geometry (void area) and "sees" also particles in the background which may also move into or out of the image plane.

- Light exposure is poor in this area which makes it difficult to identify recurring patterns in a pair of images.

- The flow is very unsteady at the flanks of the void area - at one time the flanks are static, at another particles move down (avalanche-like) very fast. In the latter case, some particles even appear only on one image and disappear on the next or at least cross more than one $\mathrm{CV}$ in one time step.

Due to these effects the measurement doesn't capture the elevated velocities and the void zone below the tubes. Thus the model cannot be validated in this region. For comparison, FIGURE 7 shows an exemplary particle assembly obtained from the DEM simulation.

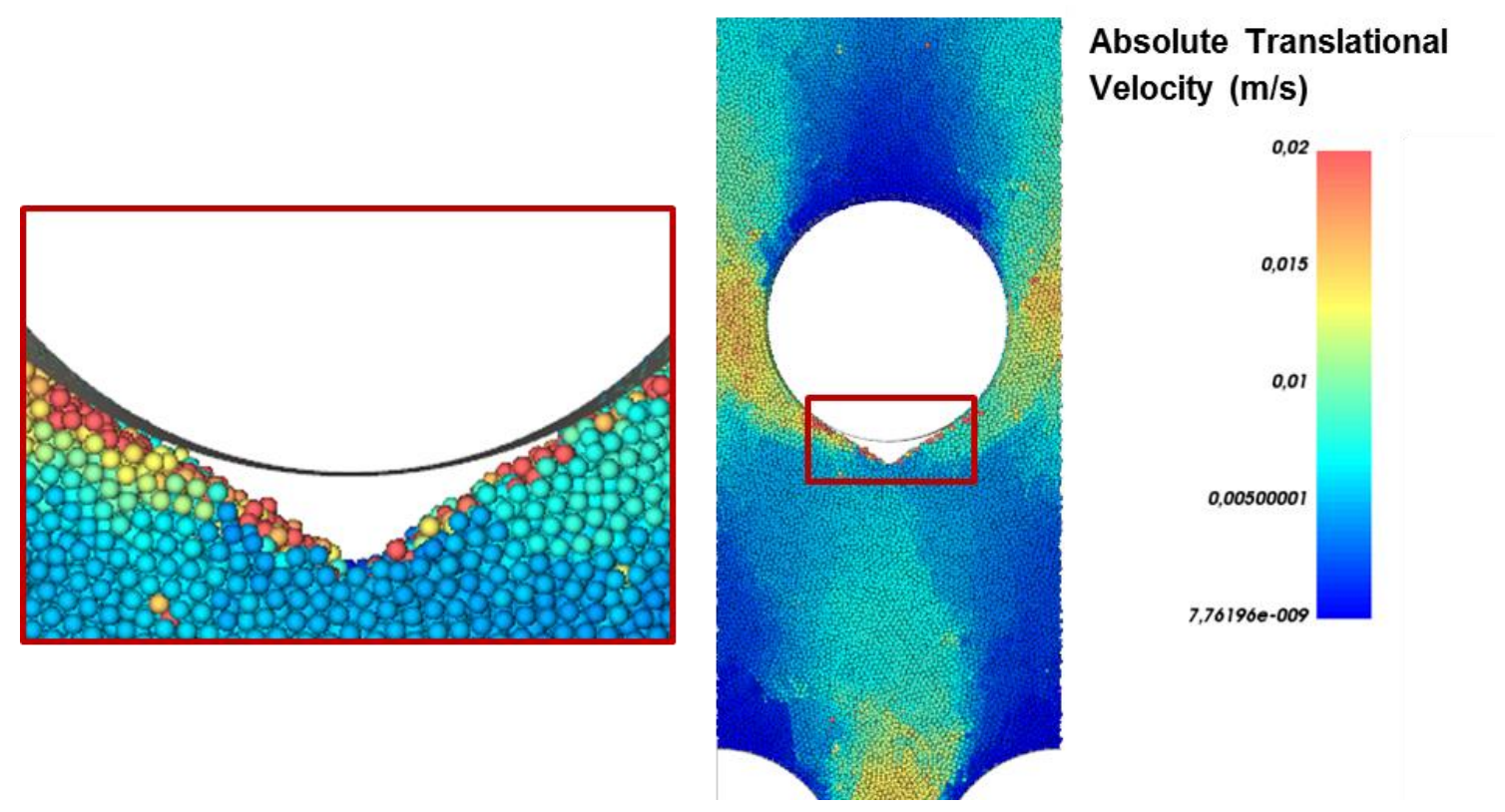

FIGURE 7. Exemplary particle assembly from DEM-simulation. Particle color according to their translational velocity. Left: Void area below the tube.

The comparison of the simulated and the measured velocity profile at the tube surface leads to the following conclusion:

In the angular range of $0^{\circ}<\omega<120^{\circ}\left(240^{\circ}<\omega<360^{\circ}\right)$ the absolute deviations of simulation and experiment are $u / u_{\mathrm{ref}}<10 \%$. They coherently yield a stagnant area $(u \approx 0)$ in the range of $\omega<50^{\circ}\left(\omega>210^{\circ}\right)$ and a maximum of flow velocity at $\omega \approx 90^{\circ}$.

In the range of $120^{\circ}<\omega<240^{\circ}$ significant deviations between simulation and experiment are observed directly at the tube surface due to the formation of a void area. The deviations diminish with increasing distance from the tube surface. At a distance of four particle diameters the absolute deviations are $u / u_{\text {ref }}<10 \%$. 


\subsection{Influence of the front wall on the flow pattern}

Many researchers have found granular materials to move as a "plug-flow" in different experimental setups in the past (e.g. [9] [43] [11]). In case of a plug-flow the velocity of the flow is assumed to be constant across the flow cross-section. Applying this assumption in the direction of the tubes axes, i.e. z-direction, suggests that the flow profile inside the bulk virtually inaccessible for measurements - should be the same as the one directly visible from the acrylic glass front.

To check whether this assumption holds true for the case at hand we now use the validated DEM model to predict the inner flow field. FIGURE 8 shows two contour plots: The plot on the left corresponds to the simulation data presented in the preceding sections and displays the flow at a front wall with $\mu_{\text {front }}=0.5$. The plot on the right displays the flow inside the bulk excluding the effect of confining walls $\left(\mu_{\text {front }}=0.0\right)$.
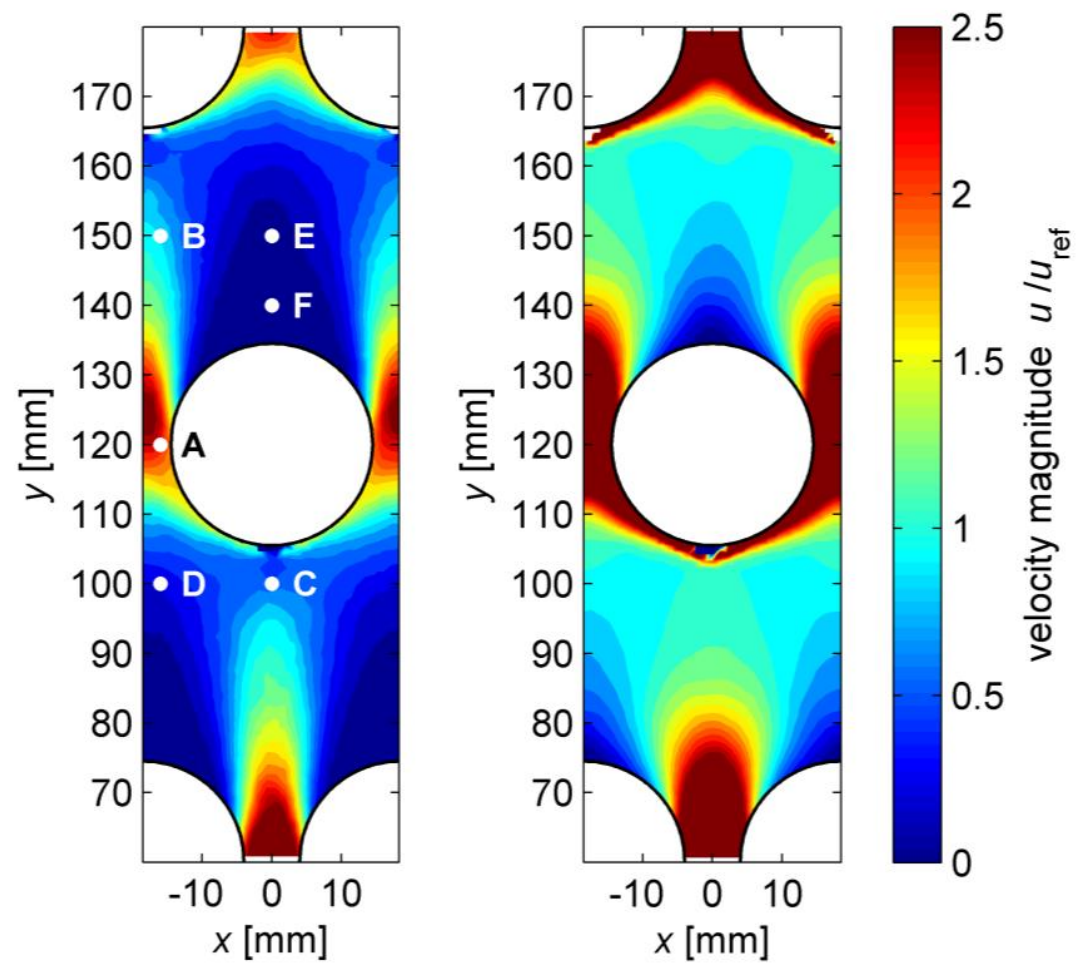

FIGURE 8. Contour plots of granular flow speed obtained from DEM simulations. Left: Flow at the front wall taking wall friction into account $\left(\mu_{\text {wall }}=0.5\right)$. Right: Flow inside the bulk.

At the front wall the flow is considerably slower and the "static area" above the tube is much bigger than inside the bulk. The influence of the void area, leading to elevated flow speeds below the tube, increases inside the bulk. Thus, the plug-flow assumption is not valid in the considered case. Furthermore, the influence of the front wall is well captured by including the measured friction coefficient in the model.

FIGURE 9 shows velocity profiles perpendicular to the transparent front wall at different position A-F as marked in FIGURE 8. At a distance of 15 particle diameters $(9 \mathrm{~mm})$, the profiles A-E reach $97 \%$ of the maximum velocity (of the corresponding profile). For profile $\mathrm{F}$, which is located inside the stagnant zone, it is $90 \%$ of the maximum velocity. Thus, for the heat transfer in engineering scale devices the impact of the wall should be negligible. 


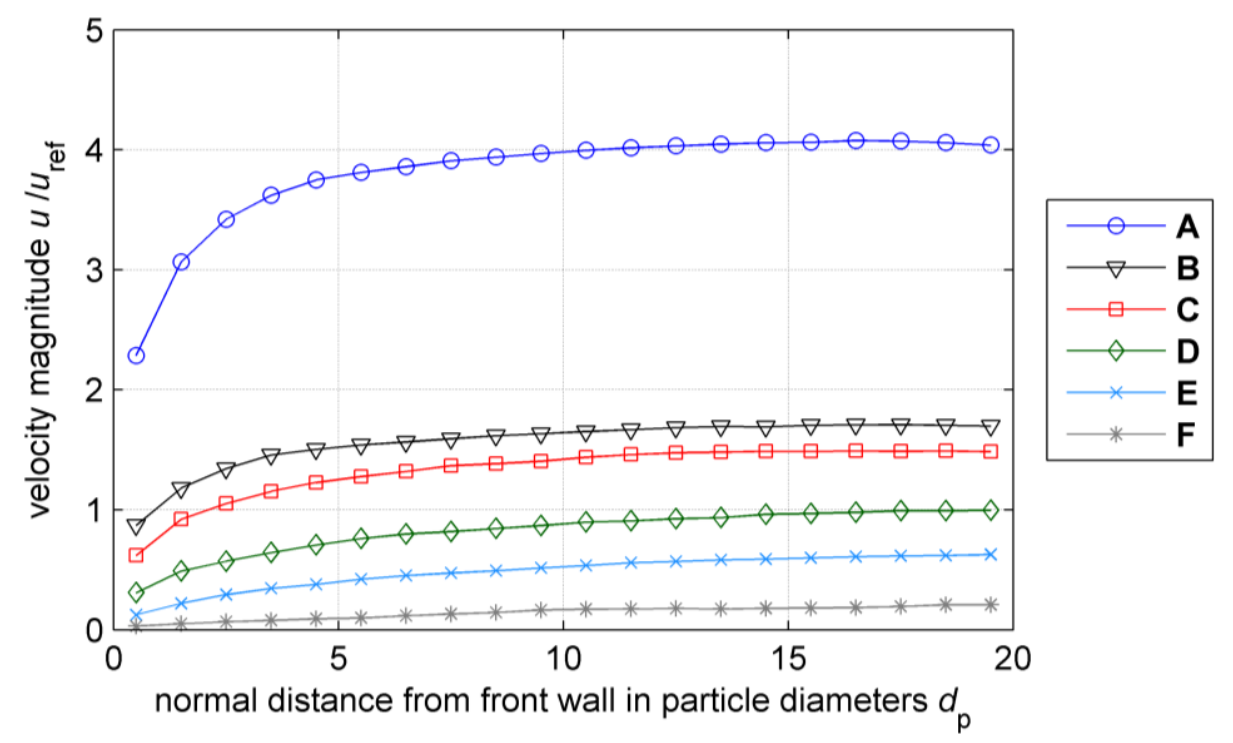

FIGURE 9. Velocity profiles in z-direction (perpendicular to the transparent front wall). The locations A-F are marked in FIGURE 8.

\section{Summary and conclusions}

Granular materials exhibit several beneficial properties with respect to application as heat transfer fluid in solar thermal power plants. To this end, particle heat exchangers are needed to efficiently discharge thermal energy from hot bulk materials. Moving bed heat exchangers are a favorable design option in this application. However, the complex nature of the dense granular flow in such heat exchangers calls for new design tools to precisely calculate the thermal performance of the device.

In this study we introduce a numerical model utilizing the discrete element method (DEM) to calculate the (isothermal) granular flow around the horizontally arranged tubes in a moving bed heat exchanger. The simulations are compared to experimental data obtained from PIV measurements.

We compare simulation and experiment by means of velocity profiles along horizontal section planes and the velocity profile along the surface of the tube. Simulation and experiment show good agreement with deviations below $15 \%$ of the reference velocity $\left(|\Delta u| / u_{\text {ref }}<0.15\right)$ in the upper part of the tube (up to a position angle of $\omega \approx 120^{\circ}$ ). Only in specific areas increased deviations $\left(|\Delta u| / u_{\text {ref }}<0.6\right)$ are observed due to disturbing effects in the measurement data.

In the lower part of the tube directly at the tube surface, the model yields elevated velocities which are not observed in the measurements $\left(|\Delta u| / u_{\mathrm{ref}} \approx 1.0\right)$. These elevated velocities originate from the formation of a void zone below the tubes. Although the void zone and also particles with elevated velocity are also visible in the raw images of the measurement, the effects are not captured by the PIV analysis due to disturbing effects in the measurement setup, the highly dynamic flow pattern, and the inherent nature of PIV technique. This suggests that the simulation results in this area are qualitatively correct but a validation of the model in this region based on the current experimental data is not possible.

In a further step we use the validated model to assess the granular flow inside the bulk which cannot directly be inspected with measurements. It turns out that the plug-flow assumption does not hold true for the investigated geometry and that the flow pattern inside the bulk differs significantly from the one at the front wall which is accessible for measurement. Therefore, the developed model gives fundamental insight into the phenomena that determine the thermal performance of MBHE. It may be used in future works to investigate the dependence of the granular flow field on different influencing parameters such as the tube arrangement or the friction coefficients between particles and thus aiding to further promote the cost-efficiency such heat exchangers. 


\section{References}

[1] H. M. Jaeger and S. R. Nagel, "Physics of the Granular State," Science, vol. 255, pp. 1523$1531,1992$.

[2] W. Wu et al., "Proof of concept test of a centrifugal particle receiver," Energy Procedia, vol. 49, pp. 560-568, 2014.

[3] H. S. Mickley and D. F. Fairbanks, "Mechanism of Heat Transfer to Fluidized Beds," A.I.Ch.E. Journal, vol. 1, pp. 374-384, 1955.

[4] A. O. O. Denloye and J. S. M. Botterill, "Heat Transfer in Flowing Packed Beds," Chemical Engineering Science, vol. 32, pp. 461-465, 1977.

[5] W. N. Sullivan und R. H. Sabersky, „Heat transfer to flowing granular media,“ International Journal of Heat and Mass Transfer, Bd. 18, pp. 97-107, 1975.

[6] N. S. Obuskovic, Heat Transfer Between Moving Beds of Solids and a Vertical Tube, Oregon State University, 1988.

[7] J. Niegsch et al., "Heat transfer and flow of bulk solids in a moving bed," Chemical Engineering and Processing, vol. 33, pp. 73-89, 1994.

[8] H. Takeuchi, „Particles Flow Pattern and Local Heat Transfer Around Tube in Moving Bed,“ AIChE Journal, Bd. 42, pp. 1621-1626, 1996.

[9] M. Colakyan and O. Levenspiel, "Heat Transfer between Moving Bed of Solids and Immersed Cylinders," AIChE Symposium Series, vol. 80, pp. 156-168, 1984.

[10] H. Al-Ansary, A. El-Leathy and Z. Al-Suhaibani et al., "Experimental Study of a Sand-Air Heat Exchanger for Use With a High-Temperature Solar Gas Turbine Systems," Journal of Solar Energy Engineering, vol. 134, pp. 041017-1-7, 2012.

[11] N. Obuskovic, M. Colakyan and J. G. Knudsen, "Heat Transfer Between Moving Beds of Solids and a Transverse Finned Tube," Heat Transfer Engineering, vol. 12, pp. 46-52, 1991.

[12] T. Baumann, S. Zunft and R. Tamme, "Moving Bed Heat Exchangers for Use With Heat Storage in Concentrating Solar Plants: A Multiphase Model," Heat Transfer Engineering, vol. 35, pp. 224-231, 2014.

[13] D. Chehata, R. Zenit and C. Wassgren, "Dense granular flow around an immersed cylinder," AIP Physics of Fluids, vol. 15, pp. 1622-1631, 2003.

[14] A. Morris, S. Pannala and C. Hrenya, "A conductive heat transfer model for particle flows over immersed surfaces," International Journal of Heat and Mass Transfer, vol. 89, pp. 1277-1289, 2015.

[15] W.-S. Lee, S.-K. Youn and S.-I. Park, "Finite element analysis of the flow and heat transfer of solid particles in moving beds," International Journal of Energy Research, vol. 22, pp. 1145-1155, 1998.

[16] D. Gidaspow et al., "Hydrodynamics of circualting fluidized beds: Kinetic theory approach," in 7th Fluidization Conference, 1992.

[17] M. J. V. Goldschmidt, R. Beetstra and J. Kuipers, "Hydrodynamic modelling of dense gasfluidised beds: Comparison of the kinetic theory of granular flow with $3 \mathrm{D}$ hard-sphere discrete particle simulations," Chemical Engineering Scinece, vol. 57, pp. 2059-2075, 
2002.

[18] I. Goldhirsch, "Rapid Granular Flows," Annual Review of Fluid Mechanics, vol. 35, pp. 267-293, 2003

[19] K. K. Rao and P. R. Nott, An Introduction to Granular Flow, Cambridge: Cambridge University Press, 2008.

[20] J. R. Prakash and K. K. Rao, "Steady compressible flow of granular materials through a wedge-shaped hopper: the smooth wall, radial gravity problem," Chemical Engineering Science, vol. 43, pp. 479-494, 1988.

[21] S. A. Elaskar, L. A. Godoy and D. D. Gray et al., "A viscoplastic approach to model the flow of granular solids," International Journal of Solids and Structures, vol. 37, pp. 2185$2214,2000$.

[22] S. Dartevelle, "Numerical modeling of geophysical granular flows: 1. A comprehensive approach to granular rheologies and geophysical multiphase flows," Geochemistry Geophysics Geosystems, vol. 5, pp. 1-28, 2004.

[23] L. Brendel, T. Unger and D. E. Wolf, "Contact Dynamics for Beginners," in The Physics of Granular Media, Weinheim, Wiley Verlag, 2004, pp. 325-343.

[24] M. Van Zeebroeck, E. Tijskens und E. Dintwa et al., „The discrete element method (DEM) to simulate fruit impact damage during transport and handling: Case study of vibration damage during apple bulk transport,“ Postharvest Biology and Technology, Bd. 41, pp. 92100, 2006.

[25] W. R. Ketterhagen, M. T. Am Ende and B. C. Hancock, "Process Modeling in the Pharmaceutical Industry using the Discrete Element Method," Journal of Pharmaceutical Science, vol. 98, pp. 442-470, 2009.

[26] M. Paulick, M. Morgeneyer and A. Kwade, "Review on the influence of elastic particle properties on DEM simulation results," Powder Technology, vol. 283, pp. 66-76, 2015.

[27] Z. Zhou, H. Zhu and A. Yu et al., "Discrete Particle Simulation of Solid Flow in a Model Blast Furnace," ISIJ International, vol. 45, pp. 1828-1837, 2005.

[28] V. Dreissigacker, S. Zunft and H. Müller-Steinhagen, "A thermo-mechanical model of packed-bed storage and experimental validation," Applied Energy, vol. 111, pp. 1120-1125, 2013.

[29] T. Baumann and S. Zunft, "Developement and performance assessment of a moving bed heat exchanger for solar central receiver power plants," Energy Procedia, vol. 69, pp. 748$757,2015$.

[30] T. Baumann, Wärmeauskopplung aus heißen Partikelschüttungen zur Dampferzeugung, Stuttgart: Stuttgart University, 2014.

[31] D. A. Steingart and J. W. Evans, "Measurements of granular flows in two-dimensional hoppers by particle image velocimetry. Part I: experimental method and results," Chemical Engineering Science, vol. 60, pp. 1043-1051, 2005.

[32] R. J. Adrain and J. Westerweel, Particle Image Velocimetry, Cambridge: Cambridge University Press, 2011.

[33] P. A. Cundall and O. D. L. Strack, "A discrete numerical model for granular assemblies," Géothechnique, vol. 29, pp. 47-65, 1979. 
[34] M. Lätzel, From microscopic simulations towards a macroscopic description of granular media, Stuttgart University, 2003.

[35] H. Kruggel-Emden, E. Simsek, S. Rickelt, S. Wirtz and V. Scherer, "Review and extension of normal force models for the Discrete Element Methode," Powder Technology, vol. 171, pp. 157-173, 2006.

[36] O. R. Walton and R. L. Braun, "Viscosity, granular-temperature, and stress calculations for shearing assemblies of inelastic, frictional disks," Journal of Rheology, vol. 30, pp. 949980, 1986.

[37] Technical Manual Rocky 4, DEM Particle Simulator, ESSS Rocky, 2017.

[38] C. M. Wensrich and A. Katterfeld, "Rolling friction as a technique for modelling particle shape in DEM," Powder Technology, vol. 217, pp. 409-417, 2012.

[39] B. Vasarhelyi, "Some observations regarding the strength and deformability of sandstones in dry and satruated conditions," Bull. Eng. Geol. Env., vol. 62, pp. 245-249, 2003.

[40] C. J. Coetzee, "Review: Calibration of the discrete element method," Powder Technology, vol. 310, pp. 104-142, 2017.

[41] K. Senetakis, M. R. Coop and C. M. Todisco, "The inter-particle coefficient of friction at the contacts of Leighton Buzzard sand quartz minerals," Soils and Foundations, vol. 53, pp. 746-755, 2013.

[42] B. Andreotti et al., Granular Media - Between Fluid and Solid, Cambridge: Cambridge University Press, 2013.

[43] G. Tardos, "A fluid mechanistic approach to slow, frictional flow of powders," Powder Technology, vol. 92, pp. 61-74, 1997.

[44] A. Klein, D. Köneke and P.-M. Weinspach, "Schüttgutbewegung und Wärmetransport im Wanderbett - Teil 1," Chemie Ingenieur Technik, vol. 69, pp. 1130-1134, 1997.

[45] B. Andreotti et al., Granular Media - Between Fluid and Solid, Cambridge: Cambridge University Press, 2013.

[46] P. Bartsch and S. Zunft, "Granular flow around the horizontal tubes of a particle heat exchanger: DEM-simulation and experimental Comparison".submitted.

[47] T. Baumann and S. Zunft, "Developement and performance assessment of a moving bed heat exchanger for solar central receiver power plants," Energy Procedia, vol. 69, pp. 748$757,2015$.

[48] K.-W. Lim, K. Krabbenhoft and J. E. Andrade, "A contact dynamics approach to the Granular Element Method," Computer Methods in Applied Engineering, vol. 268, pp. 557$573,2014$. 